\title{
Siramesine triggers cell death through destabilisation of mitochondria, but not lysosomes
}

\author{
M Hafner Česen ${ }^{1,2}$, U Repnik ${ }^{*, 1,3}$, V Turk ${ }^{1,2}$ and B Turk ${ }^{\star, 1,4}$
}

A sigma-2 receptor agonist siramesine has been shown to trigger cell death of cancer cells and to exhibit a potent anticancer activity in vivo. However, its mechanism of action is still poorly understood. We show that siramesine can induce rapid cell death in a number of cell lines at concentrations above $20 \mu \mathrm{M}$. In HaCaT cells, cell death was accompanied by caspase activation, rapid loss of mitochondrial membrane potential (MMP), cytochrome $c$ release, cardiolipin peroxidation and typical apoptotic morphology, whereas in U-87MG cells most apoptotic hallmarks were not notable, although MMP was rapidly lost. In contrast to the rapid loss of MMP above $20 \mu \mathrm{M}$ siramesine, a rapid increase in lysosomal $\mathrm{pH}$ was observed at all concentrations tested $(5-40 \mu \mathrm{M})$; however, it was not accompanied by lysosomal membrane permeabilisation (LMP) and the release of lysosomal enzymes into the cytosol. Increased lysosomal pH reduced the lysosomal degradation potential as indicated by the accumulation of immature forms of cysteine cathepsins. The lipophilic antioxidant $\alpha$-tocopherol, but not the hydrophilic antioxidant $\mathrm{N}$-acetylcysteine, considerably reduced cell death and destabilisation of mitochondrial membranes, but did not prevent the increase in lysosomal pH. At concentrations below $15 \mu \mathrm{M}$, siramesine triggered cell death after 2 days or later, which seems to be associated with a general metabolic and energy imbalance due to defects in the endocytic pathway, intracellular trafficking and energy production, and not by a specific molecular event. Overall, we show that cell death in siramesine-treated cells is induced by destabilisation of mitochondria and is independent of LMP and the release of cathepsins into the cytosol. Moreover, it is unlikely that siramesine acts exclusively through sigma-2 receptors, but rather through multiple molecular targets inside the cell. Our findings are therefore of significant importance in designing the next generation of siramesine analogues with high anticancer potential.

Cell Death and Disease (2013) 4, e818; doi:10.1038/cddis.2013.361; published online 3 October 2013

Subject Category: Cancer

Sigma receptors were originally characterised as a subtype of the opioid receptor but were later reclassified as a novel class of receptors. ${ }^{1,2}$ There are two subtypes of sigma receptors, sigma-1 and sigma-2. ${ }^{2,3}$ Although sigma-1 receptors promote cell growth and thus inhibit cell death, ${ }^{4,5}$ activation of sigma-2 receptors was reported to trigger cell death. ${ }^{6-10}$ Sigma receptors are ubiquitously expressed in mammals with high levels found in the central nervous system (CNS) and in cancer. ${ }^{11,12}$ They are orphan receptors, exhibiting high affinity for compounds with psychotropic activity, which was explored for the development of antipsychotic drugs. ${ }^{13}$ None of the compounds made it into the clinics; however, sigma receptors remain important secondary targets in the development of antipsychotics and antidepressants with a significant potential in the treatment of drug addiction and other CNS diseases. ${ }^{14}$

Siramesine or Lu 28-179 (1'-[4-[1-(4-fluorphenyl)-1Hindol-3-yl]-1-butyl]-spiro[isobenzofuran-1(3H), 4'-piperidine]) is a sigma-2 receptor agonist that was initially developed as a potential drug for depression and anxiety, but clinical trials were abolished because of the lack of efficacy in humans. ${ }^{14,15}$ However, siramesine was found to have anticancer activity in a mouse cancer model, which revived interest in the compound. ${ }^{9}$ Siramesine has a subnanomolar affinity for sigma-2 receptors $\left(K_{d}=0.12 \mathrm{nM}\right)$ and exhibits a 140-fold selectivity for sigma-2 receptors over sigma-1 receptors $\left(K_{\mathrm{d}}=17 \mathrm{nM}\right){ }^{16-18}$ Siramesine was shown to be a potent inducer of cell death, which was linked to its potent anticancer activity..$^{8-10,19,20}$ It was initially reported to act like a lysosomotropic detergent, which in MCF-7 and WEHI-S cells induced rapid lysosome leakage and subsequent cathepsinmediated cell death independent of caspases. ${ }^{9,20}$ A similar mechanism was proposed for siramesine-mediated cell death of mast cells. ${ }^{21}$ In contrast, siramesine was demonstrated to induce caspase activation in lens epithelial cells, ${ }^{8}$ and

\footnotetext{
${ }^{1}$ Department of Biochemistry and Molecular and Structural Biology, Jožef Stefan Institute, Jamova 39, 1000 Ljubljana, Slovenia; ${ }^{2}$ Jožef Stefan's International Postgraduate School, Jamova 39, 1000 Ljubljana, Slovenia; ${ }^{3}$ Department of Biosciences, University of Oslo, Blindernveien 31 , 0371 Oslo, Norway and ${ }^{4}$ Center of Excellence CIPKEBIP, Jamova 39, 1000 Ljubljana, Slovenia

${ }^{*}$ Corresponding authors: B Turk or U Repnik, Department of Biochemistry and Molecular and Structural Biology, Jožef Stefan Institute, Jamova 39 , 1000 Ljubljana, Slovenia. Tel: + 3861477 3772; Fax: +386 1477 3984; E-mail: boris.turk@ijs.si or urska.repnik@ijs.si

Keywords: siramesine; cell death; lysosome; mitochondria; apoptosis

Abbreviations: Ac-DEVD-AFC, Ac-Asp-Glu-Val-Asp-7-amino-4-trifluoromethylcoumarin; CNS, central nervous system; E-64d, (2S, 3S)-trans-epoxysuccinylleucylamido-3-methyl-butane ethyl ester; ER, endoplasmic reticulum; LC3, microtubule-associated protein light chain 3; LMP, lysosomal membrane permeabilisation; MMP, mitochondrial membrane potential; NAC, N-acetyl-cysteine; NAO, 10-N-nonyl acridine orange; ROS, reactive oxygen species; TEM, transmission electron microscopy; Z-FR-AMC, benzyloxycarbonyl-Phe-Arg-7-amino-4-methylcoumarin; Z-VAD-FMK, benzyloxycarbonyl-Val-Ala-Asp-fluoromethylketone

Received 05.6.13; revised 18.7.13; accepted 22.7.13; Edited by G Melino
} 
benzyloxycarbonyl-Val-Ala-Asp-fluoromethylketone (Z-VADFMK) partially protected MDA-MB-435 cells against siramesine-induced cell death. ${ }^{10}$

In order to clarify the mechanism of siramesine-induced cell death, we have investigated the effect of siramesine on cell viability in several cell lines and thereafter selected HaCaT and U-87MG cells, representing two early-onset cell death modalities, to further analyse organelle integrity and caspase and cathepsin activities. Siramesine induced rapid cell death at concentrations above $20 \mu \mathrm{M}$, which was accompanied by the loss of mitochondria integrity and caspase activation and reactive oxygen species (ROS) generation. Although siramesine induced rapid increase in lysosomal $\mathrm{pH}$ at all concentrations used, the drug did not trigger lysosomal membrane permeabilisation (LMP), indicating that it does not act as a lysosomotropic detergent. At lower siramesine concentrations, cell death was observed after 2 days or later and was probably associated with a general metabolic and energy imbalance.

\section{Results}

Siramesine induces cell death in various cell lines. Initially, the effect of siramesine on cell viability was tested in different cell lines, including epithelial cell lines HaCaT,
Hsc-4, HeLa and MCF-7, neuroblastoma cell line SH-SY5Y and glioblastoma cell line U-87MG. In all cell lines, the siramesine concentration found to induce significant cell death within $8 \mathrm{~h}$ was in the range of $20-30 \mu \mathrm{M}$, whereas $90 \%$ of cells were dead after treatment with $40-50 \mu \mathrm{M}$ siramesine (Figure 1a). A pancaspase inhibitor Z-VAD-FMK largely reduced cell death in HaCaT and HeLa cells, and to a lesser extent in Hsc-4, SH-SY5Y and U-87MG cells, suggesting the involvement of caspases. Z-VAD-FMK conferred no protection to MCF-7 cells, in agreement with the absence of caspase- 3 in this cell line. In contrast, cysteine cathepsin inhibitor E-64d ((2S, 3S)-trans-epoxysuccinyl-leucylamido-3methyl-butane ethyl ester) had no effect on cell death progression in any of the cell lines, suggesting that cysteine cathepsins are not critically implicated in siramesine-induced cell death. However, the lipophilic antioxidant $\alpha$-tocopherol prevented siramesine-induced cell death very efficiently in all cell lines, in agreement with an earlier observation. ${ }^{9}$ Moreover, stepwise addition of $\alpha$-tocopherol significantly improved cell viability after prolonged incubation (Supplementary Figure 1).

Because of their different sensitivity to Z-VAD-FMK, HaCaT and U-87MG cells were selected for all subsequent studies. Lower siramesine concentrations $(<20 \mu \mathrm{M})$ did not affect viability of HaCaT cells within $8 \mathrm{~h}$ of incubation;
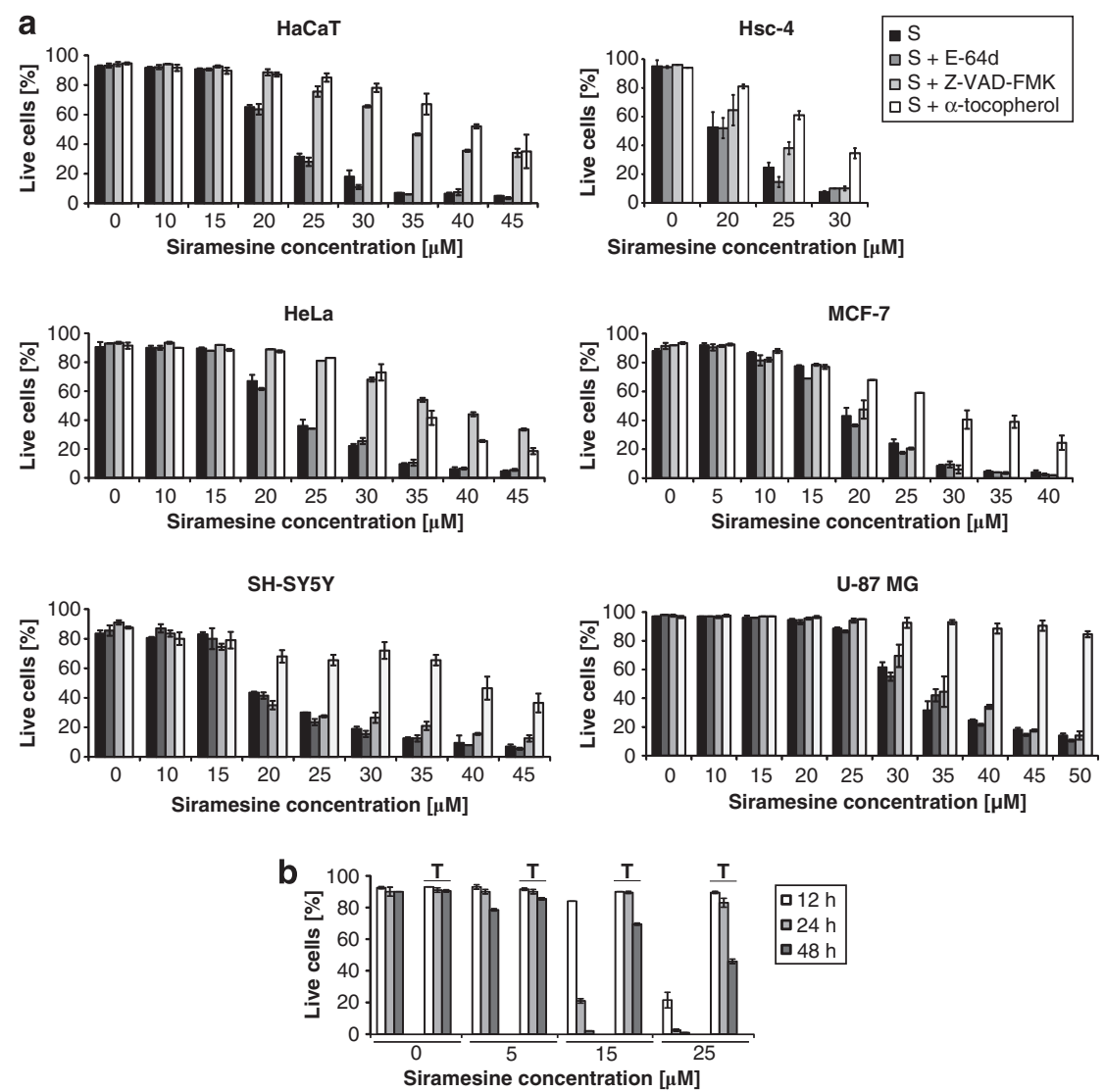

Figure 1 Effect of siramesine on viability of various cell lines. Cells were incubated with increasing concentrations of siramesine $(0-45 \mu \mathrm{M})$ for 8-48 h. The pancaspase inhibitor Z-VAD-FMK $(20 \mu \mathrm{M})$, the cathepsin inhibitor E- $64 \mathrm{~d}(10 \mu \mathrm{M})$ and the lipophilic antioxidant $\alpha$-tocopherol $(0.3 \mathrm{mM})$ were applied $2 \mathrm{~h}$ before siramesine treatment. After incubation with siramesine, the cells were stained with annexin V and propidium iodide (PI), followed by flow cytometric analysis. Live cells were defined as annexin V/PI double-negative cells. Experiments were performed in duplicate; the bars represent mean \pm S.D. (a) Effect of siramesine on viability of various cell lines after $8 \mathrm{~h}$ of incubation with siramesine. (b) Effect of lower siramesine concentrations on viability of HaCaT cells at later time points (12-48 h). S, siramesine; T, $\alpha$-tocopherol 
however, the viability decreased after prolonged incubation (48 h; Figure 1b). In the next step, caspase activity based on Ac-DEVD-AFC (Ac-Asp-Glu-Val-Asp-7-amino-4-trifluoromethylcoumarin) hydrolysis was analysed in $\mathrm{HaCaT}$ cells. Increased DEVD-ase activity was observed at all siramesine concentrations used. At higher siramesine concentrations $(\geq 25 \mu \mathrm{M})$, DEVD-ase activity was much higher and could be detected much earlier ( 4 versus $48 \mathrm{~h}$ ) compared with the low siramesine concentrations (Figure 2a). $\alpha$-Tocopherol largely prevented caspase activation at $25 \mu \mathrm{M}$ siramesine, but had less effect at $40 \mu \mathrm{M}$ siramesine (Figure 2b). Caspase activation in U-87MG cells was considerably lower than that in $\mathrm{HaCaT}$ cells and seemed to be the highest at $40 \mu \mathrm{M}$ siramesine after $6 \mathrm{~h}$. $\alpha$-Tocopherol had a minor effect on caspase activation in U-87MG cells in contrast to its major effect on cell viability. As the observed differences in DEVD-ase activity could be a consequence of different caspase levels, we next investigated whether similar
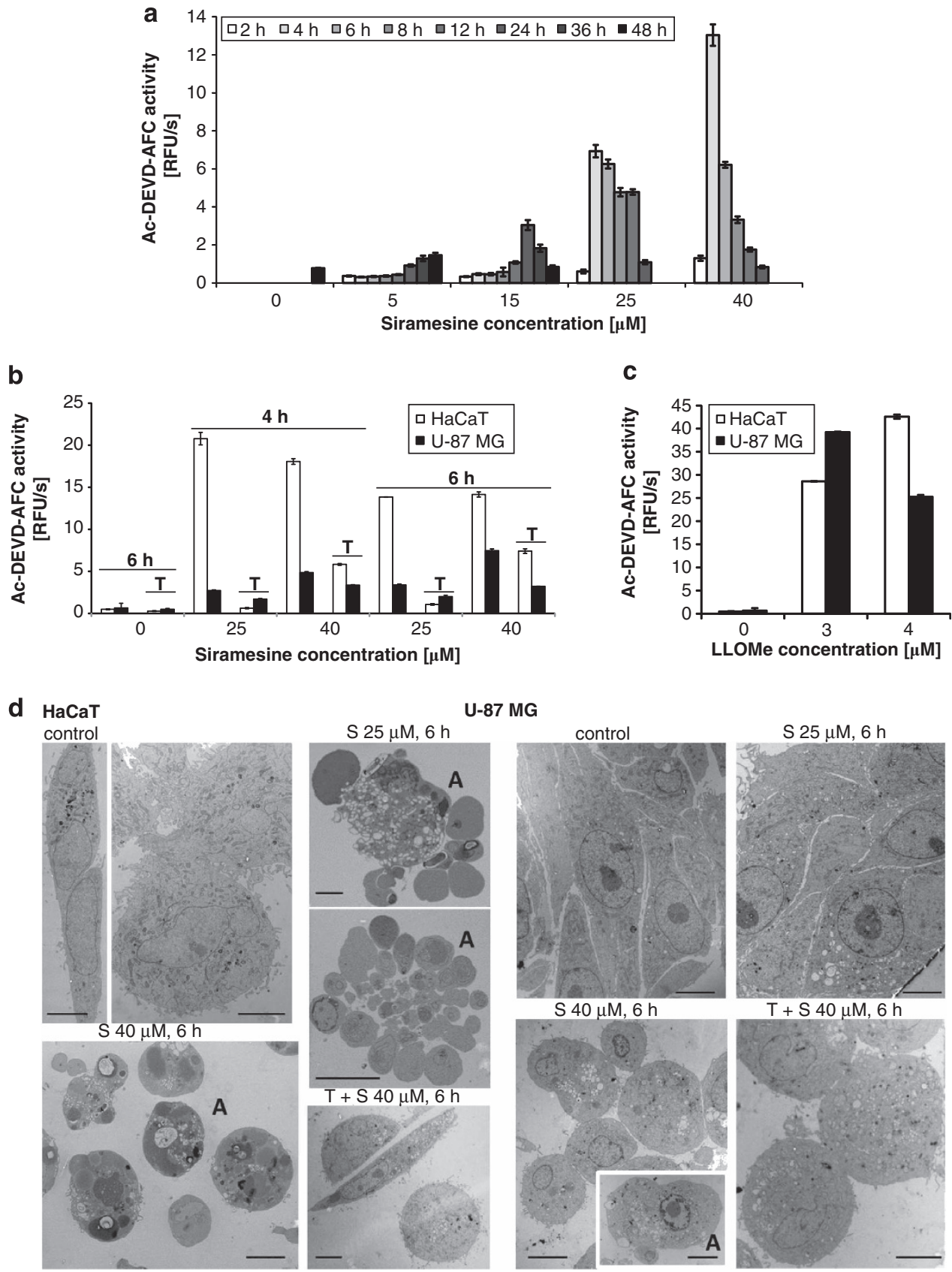

Figure 2 Siramesine activates caspases in HaCaT and in U-87MG cells. Cells were treated with different siramesine concentrations, and then the total cell lysates were collected at indicated time points. The caspase activities were measured fluorimetrically using Ac-DEVD-AFC $(10 \mu \mathrm{M})$ based on the initial velocities of substrate hydrolysis. The experiments were performed in triplicate; the bars represent mean \pm S.D. (a) DEVD-ase activity of HaCaT cells as a function of time and siramesine concentration. (b) DEVD-ase activity of HaCaT and U-87MG cells after 4- and 6-h incubation with siramesine and its prevention by $\alpha$-tocopherol. (c) DEVD-ase activity of HaCaT and U-87MG cells treated with LLOMe for $18 \mathrm{~h}$. (d) Ultrastructure of siramesine-treated HaCaT and U-87MG cells. Following the treatment, the cells were fixed with $1 \%$ glutaraldehyde and embedded in epon. Thin sections were analysed with TEM. Bar size, $5 \mu \mathrm{m}$. A, apoptotic cells; S, siramesine; T, $\alpha$-tocopherol 
differences between HaCaT and U-87MG cells would be seen using another lysosomotropic compound, LLOMe, which is known to involve caspase activation. ${ }^{22,23}$ However, DEVD-ase activity was essentially the same (Figure $2 \mathrm{c}$ ), ruling out this possibility.

Collectively, these observations suggested that siramesine can induce apoptosis, as observed in $\mathrm{HaCaT}$ cells at high siramesine concentrations, although cells can also die from different means, as seen in U-87MG cells or in HaCaT cells at low siramesine concentrations. In order to confirm different early-onset cell death modalities, we have analysed the morphology of cells by transmission electron microscopy (TEM; Figure 2d). In $\mathrm{HaCaT}$ cells treated with $40 \mu \mathrm{M}$ siramesine, some necrotic cells were observed already $1 \mathrm{~h}$ after the treatment (data not shown). Six hours post treatment with 25 or $40 \mu \mathrm{M}$ siramesine, HaCaT cells exhibited characteristic apoptotic morphology with chromatin condensation and apoptotic body formation, which was efficiently prevented by $\alpha$-tocopherol. In contrast, the shape of U-87MG cells treated with $25 \mu \mathrm{M}$ siramesine for $6 \mathrm{~h}$ appeared very similar to that of control cells. At $40 \mu \mathrm{M}$ siramesine, the cells were rounded and numerous ultrastructural changes were observed (see Figure 5 for detailed explanation), which could not be prevented with $\alpha$-tocopherol. However, the cells maintained their plasma membrane integrity, and apoptotic morphology was only observed in a minority of the cells.

Siramesine rapidly induces the loss of MMP and ROS generation. Next, we investigated the effect of siramesine on mitochondria. Siramesine concentrations $\geq 25 \mu \mathrm{M}$ decreased the mitochondrial membrane potential (MMP) in both cell lines within $15 \mathrm{~min}$, although more efficiently in HaCaT cells. Z-VAD-FMK failed to prevent the loss of MMP, suggesting that caspases act downstream of mitochondria, indicative of the intrinsic apoptotic pathway, whereas the lack of E-64d effect is consistent with no major role of cysteine cathepsins in this pathway. In contrast, $\alpha$-tocopherol was found to largely prevent the loss of
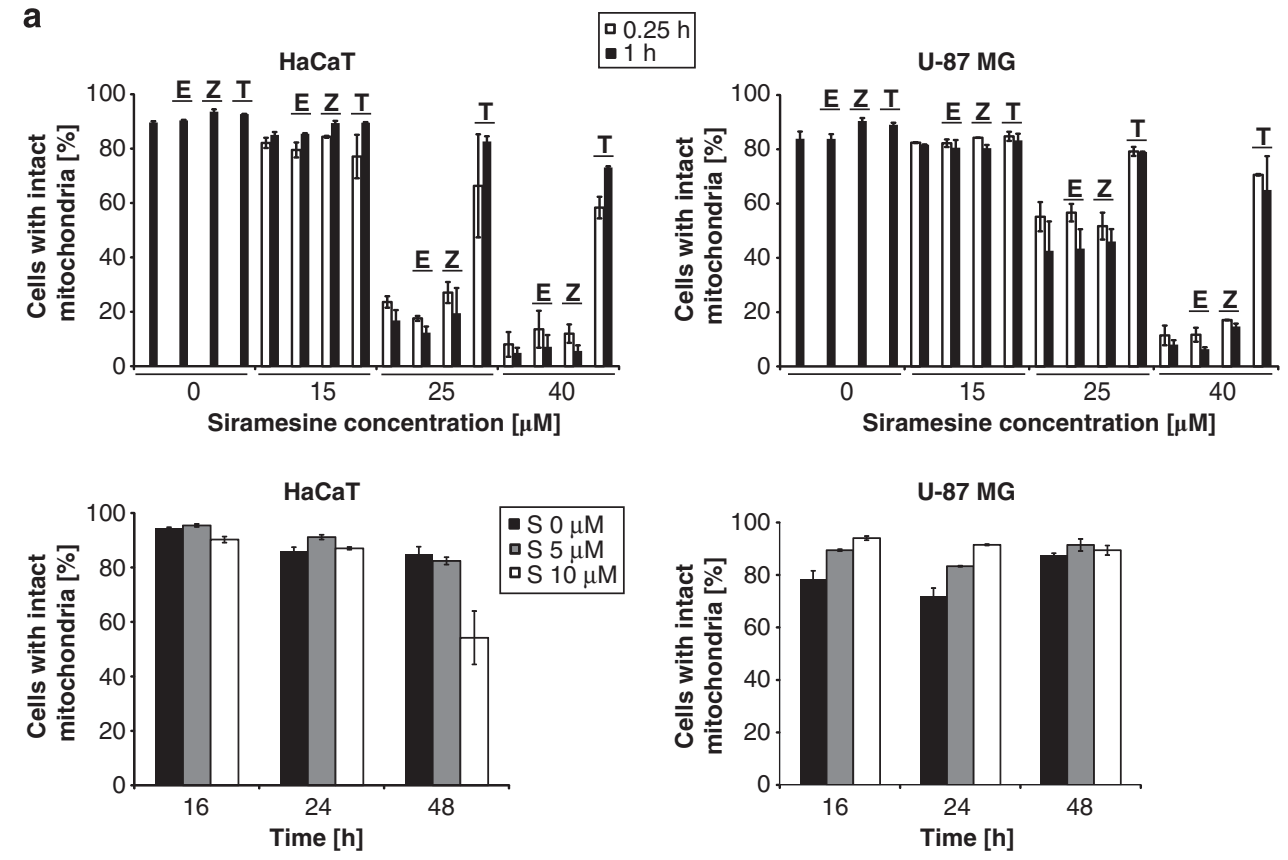

b
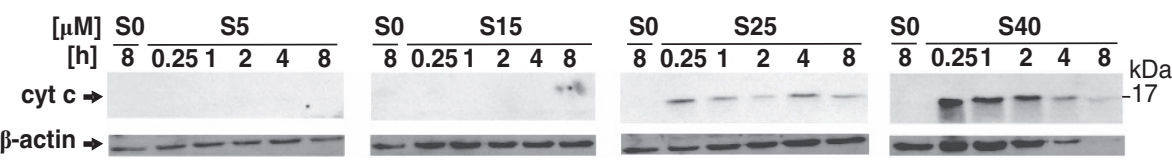

C
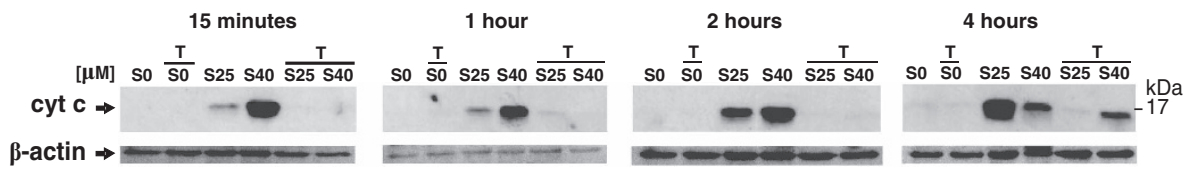

Figure 3 Effect of siramesine on mitochondria. (a) Effect of siramesine on the transmembrane potential of mitochondria. HaCaT and U-87MG cells were treated with different siramesine concentrations for the indicated periods of time. Pancaspase inhibitor Z-VAD-FMK (20 $\mu \mathrm{M})$, cathepsin inhibitor E-64d (10 $\mu \mathrm{M})$ and the lipophilic antioxidant $\alpha$-tocopherol $(0.3 \mathrm{mM})$ were applied $2 \mathrm{~h}$ before siramesine treatment. After siramesine treatment, all samples were stained at once with JC-1 at a final concentration of $3.5 \mu \mathrm{g} / \mathrm{ml}$ and analysed with a flow cytometer. Red cells were considered cells with intact mitochondria. The experiments were performed in duplicate; the bars represent mean \pm S.D. $(\mathbf{b}$ and $\mathbf{c})$ Effect of siramesine and $\alpha$-tocopherol on the release of cyt $c$ into the cytosol. After siramesine treatment, the cytosolic lysates were collected at the indicated time points followed by SDS-PAGE, western blotting and immunodetection of cyt c. Z, Z-VAD-FMK; E, E-64d; T, $\alpha$-tocopherol; S, siramesine; cyt c, cytochrome $c$ 
MMP (Figure 3a). However, at siramesine concentrations $\leq 10 \mu \mathrm{M}$, most cells retained their MMP until $48 \mathrm{~h}$ after the treatment (Figure 3a).

On the basis of 10-N-nonyl acridine orange (NAO) staining, siramesine was also found to induce cardiolipin peroxidation accompanied by major changes in the structural integrity of the mitochondria, which could be largely prevented by $\alpha$-tocopherol (Supplementary Figure 2). Furthermore, in HaCaT cells higher siramesine concentrations induced cytochrome $c$ release into the cytosol within $15 \mathrm{~min}$, which is consistent with triggering the intrinsic apoptotic pathway (Figure 3b). Again, $\alpha$-tocopherol largely prevented the release of cytochrome $c$ (Figure $3 c$ ). Within the first $15 \mathrm{~min}$ of incubation with siramesine, significant amounts of ROS were generated, especially at high concentrations of siramesine, which were efficiently blocked by $\alpha$-tocopherol (Figures $4 a$ and $b$ ). At lower siramesine concentrations $(10 \mu \mathrm{M})$, the peak of ROS generation was observed at a later time point ( $8 \mathrm{~h}$; Figure $4 \mathrm{c}$ ). As $\alpha$-tocopherol is not just an antioxidant, but as a lipophilic compound can also insert into the membrane thereby protecting it against oxidation and other types of damage such as PLA2 hydrolysis, ${ }^{24}$ we tested the idea that the protective effect against siramesine action is not linked with its antioxidant function, but is rather a consequence of membrane stabilisation. Therefore, we have used another antioxidant, $\mathrm{N}$-acetyl-cysteine (NAC), which is efficiently scavenging ROS in a number of experimental conditions already at concentrations below $1 \mathrm{mM} .{ }^{25}$ However, NAC was neither able to block viability nor protect mitochondria from the loss of MMP (Figure 4d), suggesting that the protective effect of $\alpha$-tocopherol could be largely attributed to the mitochondrial membrane stabilisation and that ROS generation is of minor importance and likely to be a consequence of the loss of MMP.

Siramesine induces major ultrastructural changes in cells. The ultrastructural changes in cells treated with siramesine were also monitored with TEM. In addition to the apoptotic changes observed in the siramesine-treated $\mathrm{HaCaT}$ cells (Figure 2d), some damaged mitochondria and enlarged vacuoles containing cytoplasmic inclusions that could represent autophagic structures were observed in nonapoptotic cells treated with $\alpha$-tocopherol and siramesine (Figure 5). Moreover, numerous vacuoles with empty lumen were seen in the siramesine-treated U-87MG cells, with or without $\alpha$-tocopherol pretreatment. A comparison between different samples of U-87MG cells suggests that these vacuoles arise from Golgi cisternae and vesicles.
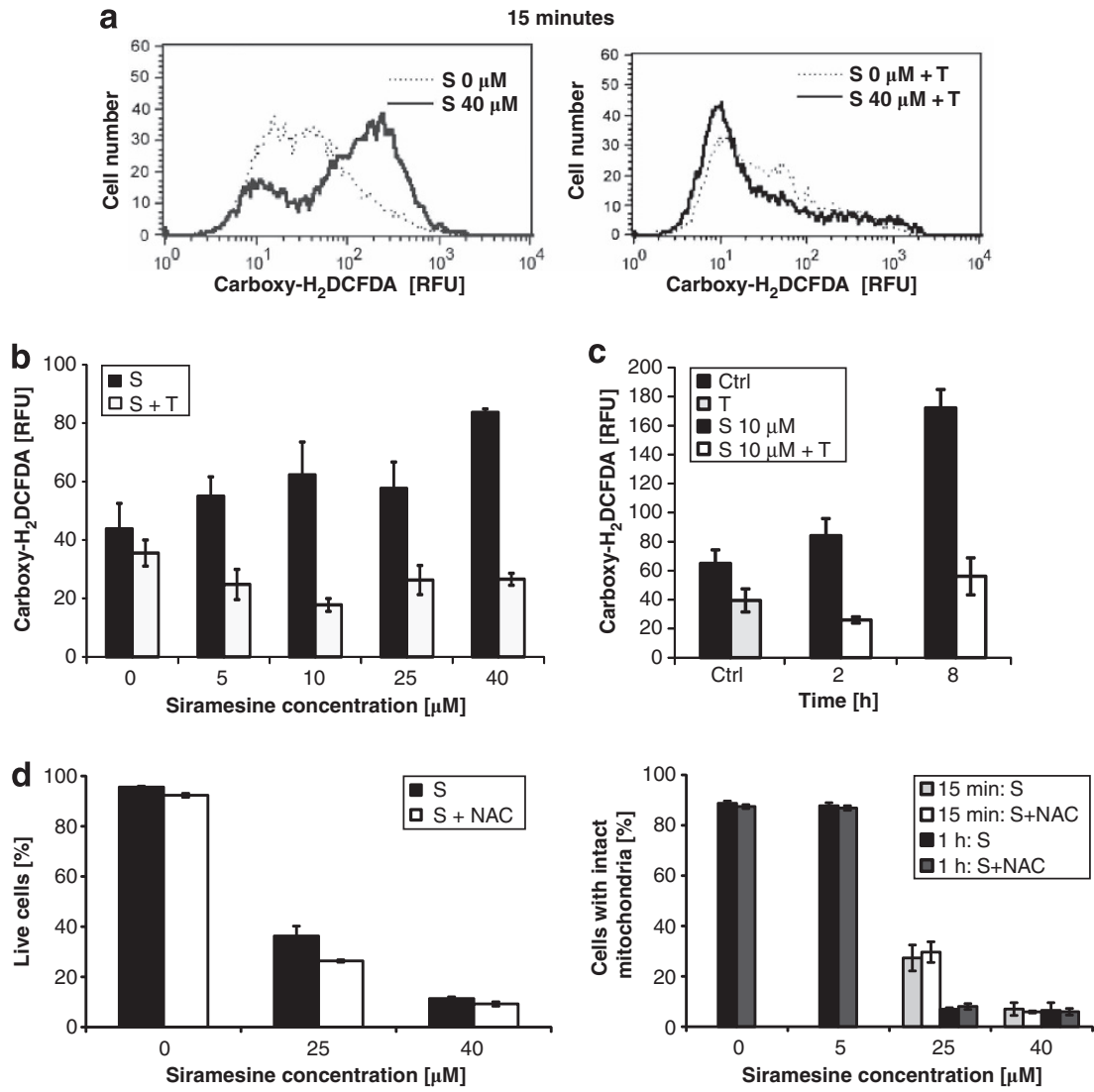

Figure 4 ROS generation in siramesine-induced cell death. HaCaT cells were treated with different siramesine concentrations. Antioxidants $\alpha$-tocopherol ( $0.3 \mathrm{mM})$ and NAC $(8 \mathrm{mM})$ were applied $2 \mathrm{~h}$ before siramesine treatment. After siramesine treatment, carboxy- $\mathrm{H}_{2} \mathrm{DCFDA}$ was added to all samples at once and analysed with a flow cytometer. The geometric mean of carboxy- $\mathrm{H}_{2} \mathrm{DCFDA}$ fluorescence intensity for each sample is displayed. The experiments were performed in duplicate; the bars represent mean \pm S.D. (a) A histogram after 15 min incubation with $40 \mu \mathrm{M}$ siramesine in the presence or absence of $\alpha$-tocopherol. Solid lines represent siramesine-treated cells; dashed lines represent control cells. (b) ROS generation 15 min after the treatment with different siramesine concentrations. (c) ROS generation $2 \mathrm{~h}$ and $8 \mathrm{~h}$ after treatment with a low concentration of siramesine $(10 \mu \mathrm{M})$. (d) The effect of NAC on viability and MMP. S, siramesine; T, $\alpha$-tocopherol 

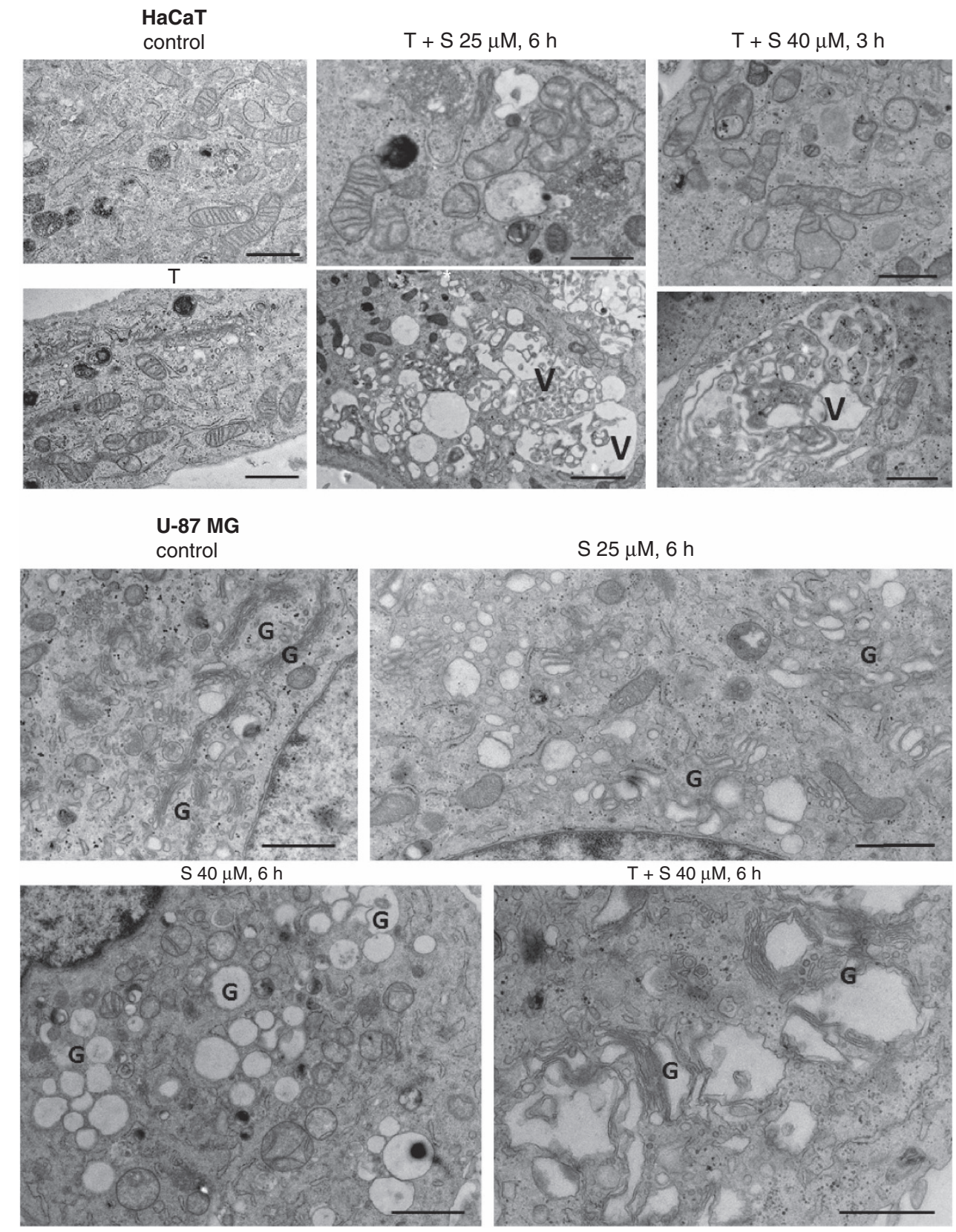

$\mathrm{S} 25 \mu \mathrm{M}, 6 \mathrm{~h}$
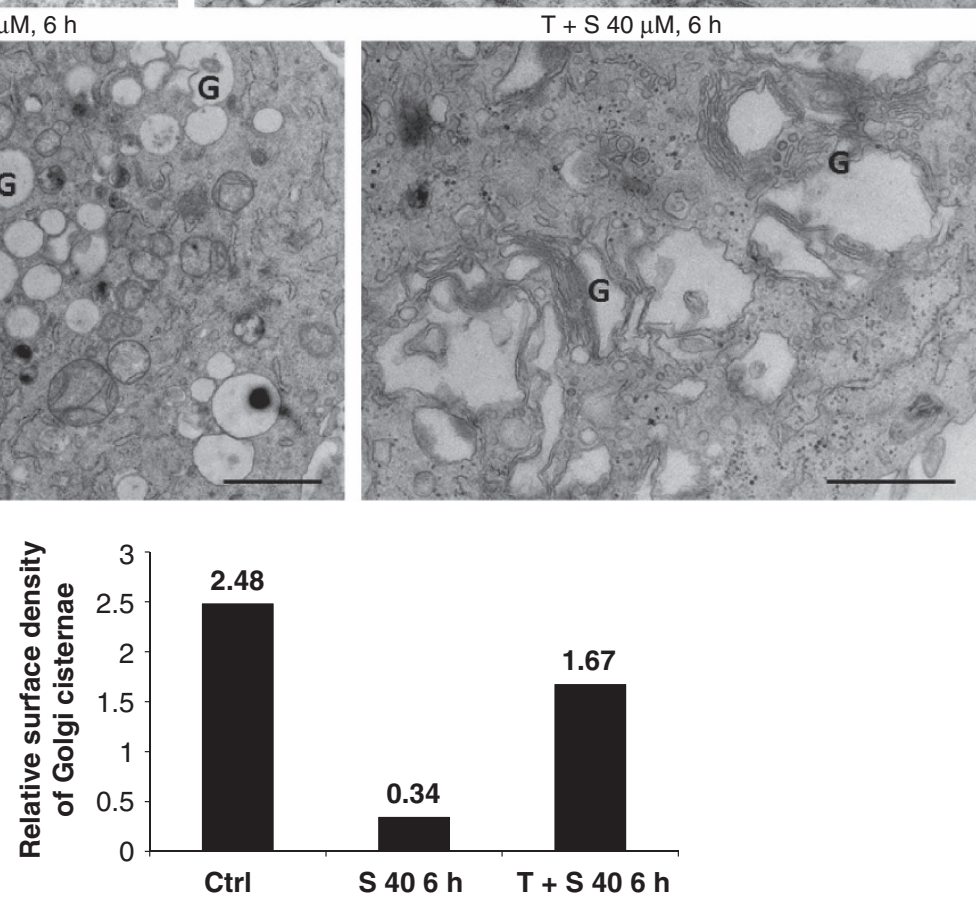

Figure 5 Ultrastructure of siramesine-treated $\mathrm{HaCaT}$ and U-87MG cells by TEM. Following the treatment, cells were fixed with $1 \%$ glutaraldehyde and embedded in epon. Thin sections were analysed with TEM. The presence of Golgi cisternae in U-87MG cells in thin sections was quantified using stereology. Bars present ratios between the total length of the Golgi cisternal membrane (estimated by intersection counting) and the total cytoplasmic area (estimated by point counting). Bar size, $1 \mu \mathrm{m}$. V, vacuole with cytoplasmic inclusions; G, area with Golgi cisternae and vesicles; S, siramesine; T, $\alpha$-tocopherol 
Stereological analysis of the presence of the Golgi cisternae confirmed their reduction in siramesine-treated cells (Figure 5). In control untreated cells, there were prominent stacks of elongated narrow Golgi cisternae, whereas in cells treated with siramesine Golgi cisternae appeared enlarged and lost the characteristic shape. Swelling and vacuolisation of the Golgi cisternae and vesicles were most notable at higher siramesine concentrations and could be reduced, but could not be prevented with $\alpha$-tocopherol. In HaCaT cells, modifications of the Golgi cisternae could also be noticed but appeared less prominent (Figure 5). In thin Tokuyasu sections of the U-87MG cells, some of the enlarged vacuoles were labelled for LAMP-1, representing endosomes/lysosomes, whereas some were not labelled and probably corresponded to the enlarged Golgi cisternae observed in epon sections (Supplementary Figure 3). In addition, in U-87MG cells we observed abundant glycogen grains in control cells and cells treated with $\alpha$-tocopherol and siramesine, but not in cells treated only with siramesine, suggesting that siramesine-treated cells rely on glycolysis for the production of energy, presumably because of non-functional mitochondria (Supplementary Figure 6c and Figure 5).

Siramesine-induced cell death is not mediated by LMP. Next, the effect of siramesine on lysosomes was investigated, as earlier reports suggested that siramesine induces LMP and consequently the leakage of lysosomal cathepsins, which are believed to be essential for siramesine-mediated cell death. ${ }^{9,20}$ HaCaT and U-87MG cells were stained with LysoTracker Green, which accumulates in acidic vesicles, and analysed by flow cytometry. Within $15 \mathrm{~min}$ after siramesine treatment, most cells completely lost the ability to retain LysoTracker Green regardless of the siramesine concentration used (Figure 6a). This could not be prevented with protease inhibitors Z-VAD-FMK or E-64d, or with $\alpha$-tocopherol (Figure 6a).

The loss of acidic vesicles could be explained either by LMP or by an increase in lysosomal pH independent of LMP. To evaluate the induction of LMP, the activity of cysteine cathepsins after selective plasma or total cell membrane lysis with digitonin was assayed using the general cathepsin substrate benzyloxycarbonyl-Phe-Arg-7-amino-4-methylcoumarin (Z-FR-AMC; Figure 6b). Initially, the digitonin concentration required for selective plasma cell membrane lysis was optimised in HaCaT cells (Supplementary Figure 4a). In agreement with previous results, ${ }^{26}$ cysteine cathepsin activities measured at digitonin concentrations up to $30 \mu \mathrm{g} / \mathrm{ml}$, which disrupt only the plasma membrane, were $<10 \%$ of the total cathepsin activity, measured at $200 \mu \mathrm{g} / \mathrm{ml}$ digitonin, which also disrupts organelle membranes. No major increase in cysteine cathepsin activity after plasma membrane lysis was observed in HaCaT and U-87MG cells treated with siramesine in comparison with control cells, regardless of the siramesine concentration or incubation period used, suggesting that siramesine did not induce LMP, not even after prolonged incubation (Figure 6b). In addition, $\alpha$-tocopherol had no effect on cysteine cathepsin activity, in agreement with the absence of LMP (Supplementary Figure $4 \mathrm{~b}$ ). To verify this result, a putative release of cathepsin $L$ into the cytosol was examined by immunological detection in the extracts of $\mathrm{HaCaT}$ cells prepared after 1-h incubation with siramesine. Cathepsin L could only be detected in total extracts of control cells prepared with $200 \mu \mathrm{g} / \mathrm{ml}$ digitonin, consistent with the absence of LMP (Figure 6c). Furthermore, similar results were obtained for another lysosomal enzyme, $\mathrm{N}$-acetyl- $\beta$-D-glucosaminidase, confirming that the lysosomes were not permeabilised even after $7 \mathrm{~h}$ of incubation with siramesine at concentrations below $20 \mu \mathrm{M}$ (Supplementary Figure 4c). Taken together, these results indicate that the reduced ability of siramesine-treated cells to accumulate LysoTracker Green is not due to LMP.

Siramesine prevents autophagosome fusion with lysosomes. In order to analyse whether the increase in $\mathrm{pH}$ affected normal cathepsin function and thus the autophagic flux, the total cell lysates of HaCaT cells were analysed for the protein levels of cysteine cathepsins and the autophagic marker microtubule-associated protein light chain 3 (LC3). ${ }^{27}$ Significant accumulation of the proforms of cysteine cathepsin L (Figure 7a) and cathepsins B and C (Supplementary Figure 5) was observed upon treatment with siramesine concentrations $\leq 15 \mu \mathrm{M}$, suggesting the synthesis of new cathepsin molecules. However, no processing to the mature forms was observed, suggesting that proteolysis in the endosomes/lysosomes was impaired. This is also consistent with the accumulation of the LC3-II form with time. As shown in Figure 7a, only LC3-I was present in the control sample, whereas in siramesine-treated cells a band for LC3-II appeared $30 \mathrm{~min}$ after the treatment and increased over time, regardless of the siramesine concentration. This further suggests that the accumulation of LC3 molecules at later time points and at siramesine concentration $\leq 15 \mu \mathrm{M}$ was due to protein synthesis. In cells treated with siramesine concentration above $20 \mu \mathrm{M}$ there was less LC3-II, and the band for LC3-I disappeared, which is probably a consequence of inhibited protein synthesis during apoptosis. Finally, immunocytochemistry results showed that the majority of the LC3-positive vesicles did not colocalise with the LAMP-2-positive vesicles (Figure 7b). This further suggests that siramesine not only inhibits the turnover of LC3 due to reduced endosomal/ lysosomal degradation but also affects the fusion of autophagosomes with endosomes/lysosomes, thereby decreasing the autophagic flux.

Siramesine-mediated cell death is independent of endoplasmic reticulum. Finally, we also tested the integrity of the endoplasmic reticulum (ER) membrane after siramesine treatment, as sigma-2 receptors were suggested to be localised on the ER. ${ }^{28}$ Free intracellular calcium was measured using Fluo-4 dye. Ionomycin, which acts as an ionophore, was used as a positive control, and the calcium chelator EGTA was used as a negative control. Not even the highest siramesine concentration $(40 \mu \mathrm{M})$ was able to trigger permeabilisation of the ER membrane within 30 min after the treatment (Supplementary Figure 6a), suggesting that siramesine does not act through the ER. In U-87MG cells treated with $40 \mu \mathrm{M}$ siramesine for $6 \mathrm{~h}$, swelling of the ER cisternae was observed in some cells (Supplementary Figure 6b). However, it was unlikely a direct effect of siramesine, but rather suggesting that cells were losing 
their ability to sustain homeostasis due to defective energy metabolism.

\section{Discussion}

Because of a significant potential of sigma-2 receptors in cancer treatment, the development of new sigma-2 receptor agonists is rapidly progressing and a number of novel compounds have recently been synthesised. ${ }^{29,30}$ However, the mechanism of siramesine-induced cell death, which is of major importance for future development of the field, is still not clear.

Here we have shown that siramesine induces cell death at low micromolar concentrations in a number of cell lines of
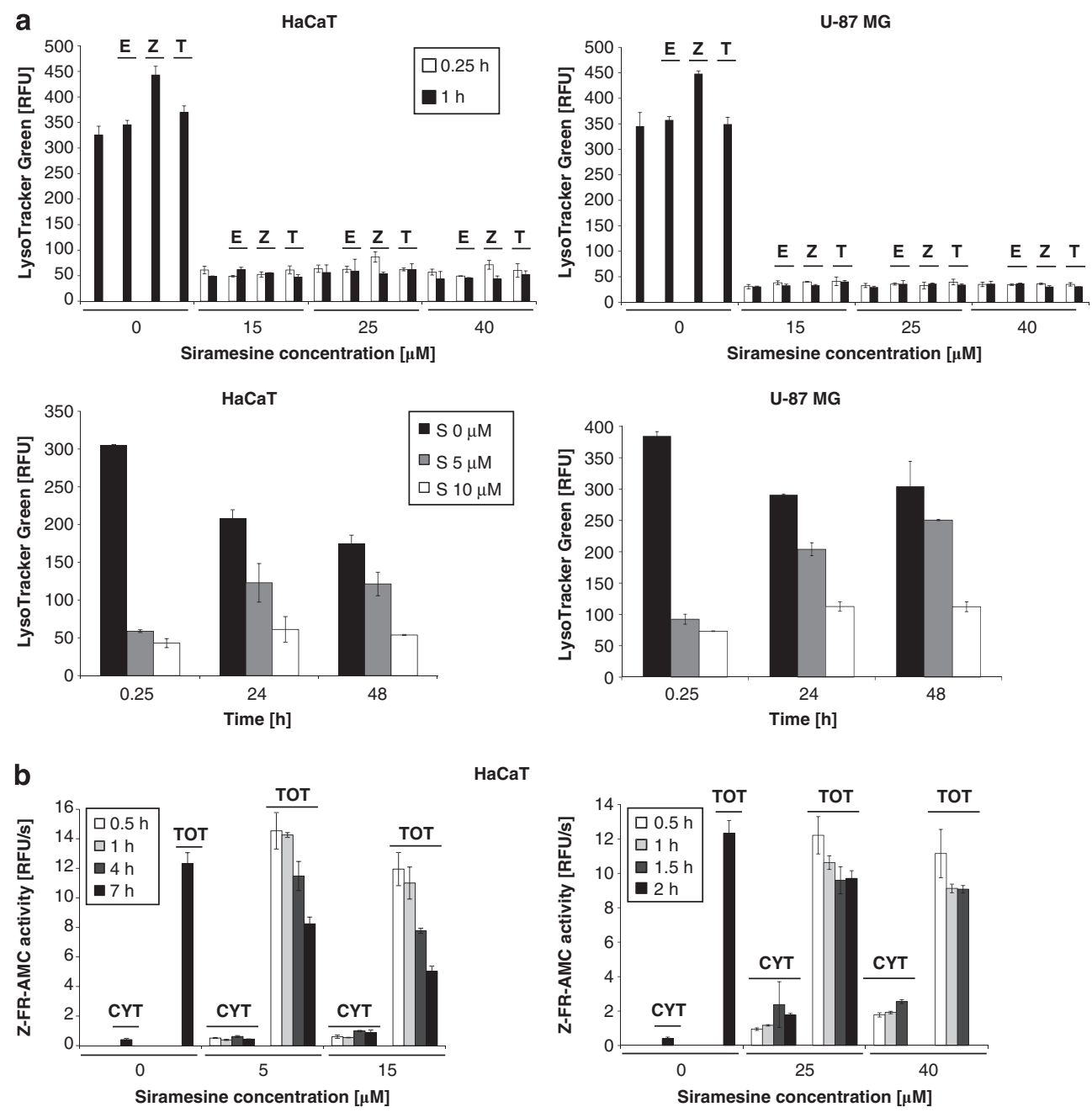

HaCaT

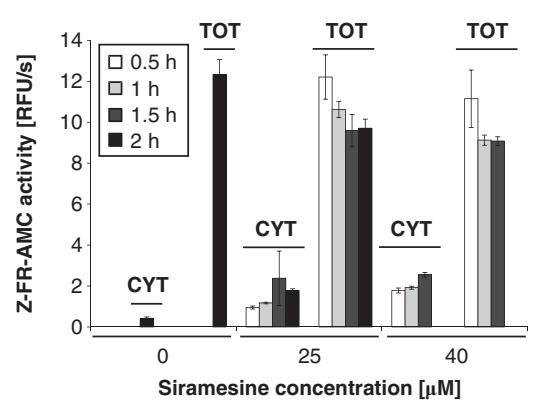

U-87 MG
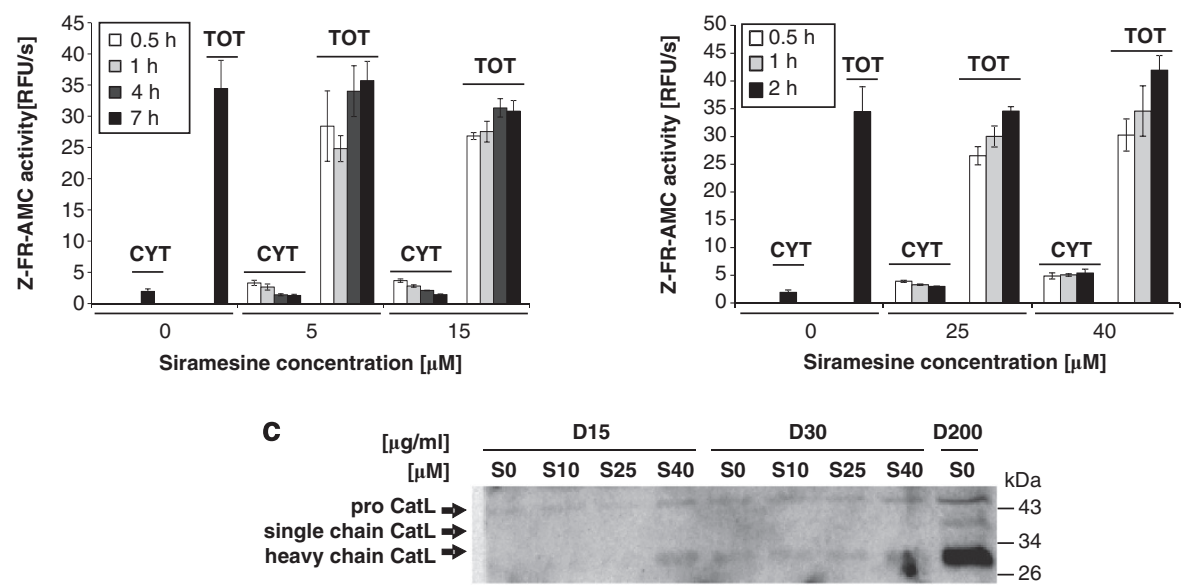
different origin and that the mitochondria, and not lysosomes, are critical organelles for the induction of cell death. The rapid loss of MMP induced by siramesine was accompanied by a rapid generation of ROS already $15 \mathrm{~min}$ post treatment. Although siramesine-induced ROS generation was observed previously, it was only shown to occur after $4 \mathrm{~h} .{ }^{9}$ However, a critical role of ROS in siramesine-induced cell death is unlikely. Although $\alpha$-tocopherol reduced not only ROS generation but also cell death, caspase activation, loss of MMP and ultrastructural changes, another antioxidant, NAC, had no protective effect on cell viability or on the loss of MMP. Nevertheless, ROS generation probably enhances cell death signalling and an important target of ROS in the mitochondrial membrane is cardiolipin, whose peroxidation was shown to enable oxidative stress-mediated cytochrome $c$ release from mitochondria. ${ }^{31}$ The interaction of siramesine with mitochondrial membranes seems to be sufficient to functionally disable mitochondria and affect cell homeostasis in the long run, thereby initiating cell death. However, it is unclear whether siramesine affects the functional integrity of the mitochondrial membrane directly or indirectly through destabilising its structural integrity.

Among the first intracellular targets of siramesine are the acidic vesicles, including endosomes and lysosomes. However, our results demonstrate that siramesine only induced a rapid increase in $\mathrm{pH}$ but not LMP, and a release of cathepsins into the cytosol, arguing against siramesine as a lysosomotropic detergent, unlike LLOMe. ${ }^{22,23}$ As the release of cathepsins into the cytosol is required for their active role in cell death signalling, ${ }^{32-34}$ this explains why siramesine cannot trigger cell death through lysosomal cathepsins. This is further supported by the lack of protective effect of the cathepsin inhibitor E-64d on cell viability and organelle damage. Moreover, $\alpha$-tocopherol, which had a significant effect on cell viability, loss of MMP and ROS generation, had no effect on the increase of lysosomal $\mathrm{pH}$ and on cathepsin activity measured to test lysosomal membrane structural integrity (Repnik et al., unpublished). These results are in apparent disagreement with earlier studies, which suggested a detergent mode of siramesine action. ${ }^{8,9,20,21}$ However, previous studies have not measured cathepsin activity directly on permeabilised cells, but rather on the isolated light membrane fraction and at very late time points (12 $\mathrm{h}$ post treatment). Alternatively, the release of cathepsins into the cytosol was analysed by whole-cell immunofluorescence, which can be difficult to reliably interpret due to limitations in the specificity of antibodies and the sensitivity of the fluorescent signal detection, or only indirectly based on a partial protective effect of E-64d.

As demonstrated, siramesine affects several intracellular processes, which is summarised in Figure 8 . It seems unlikely that the molecular mechanisms of siramesine action are linked solely to the sigma-2 receptors, as initially suggested. 4,6,7,9-12,35 The siramesine concentrations required to kill cells in some of these earlier studies were in the low micromolar range, which is more than 10000 -fold higher than the $K_{d}$ value for siramesine binding to the sigma-2 receptors, ${ }^{16-18}$ which strongly argues against the idea of siramesine only signalling through sigma-2 receptors. Furthermore, lowmicromolar siramesine concentrations also exceed the $K_{d}$ value of siramesine for sigma- 1 receptors by several 100 -fold, suggesting that under these conditions also the sigma-1 receptors would be completely saturated, thereby protecting cells against cell death. ${ }^{4,5}$ Moreover, siramesine was shown to interact with phospholipids with a dissociation constant of $\sim 0.23 \mu \mathrm{M},{ }^{36}$ which is in the range where siramesine induces cell death. This is in agreement with our finding that the protective effect of $\alpha$-tocopherol is a consequence of mitochondrial membrane stabilisation. As siramesine can also interact with phospholipids, ${ }^{36}$ it can probably structurally destabilise mitochondrial membranes through interaction with phosphatidic acid, which modulates the curvature of membranes, ${ }^{37}$ or with cardiolipin. ${ }^{38}$ In addition, phosphoinositides are essential regulators of intracellular membrane trafficking and their interaction with siramesine may affect intracellular trafficking as suggested by vacuolated Golgi cisternae and largely reduced autophagosome-lysosome fusion. Moreover, ligands other than sigma receptors have been identified for the related piperidine analogues, such as the siramesine homologue haloperidol, which has been found to directly inhibit various types of ion channels, including the ATPsensitive potassium channels. ${ }^{39}$ Similar effects were also observed for other sigma-2 receptor agonists, ${ }^{40,41}$ suggesting that siramesine may inhibit vacuolar V-ATPase in the endosomal/lysosomal membrane, resulting in an extremely rapid increase in $\mathrm{pH}$ in siramesine-treated cells. This further suggests that siramesine may inactivate even the structurally similar F-type ATPase in the mitochondrial membrane, ${ }^{42,43}$ thereby contributing to the loss of mitochondria energy production.

Siramesine concentrations used in our study $(5-40 \mu \mathrm{M})$ surpassed the range of siramesine concentrations used in

Figure 6 Effect of siramesine on lysosomes. (a) The ability of lysosomes to accumulate LysoTracker Green after siramesine treatment. HaCaT and U-87MG cells were treated with different siramesine concentrations and incubated for the indicated time. Pancaspase inhibitor Z-VAD-FMK $(20 \mu \mathrm{M})$, cathepsin inhibitor E-64d (10 $\mu \mathrm{M})$ and the lipophilic antioxidant $\alpha$-tocopherol $(0.3 \mathrm{mM})$ were applied $2 \mathrm{~h}$ before siramesine treatment. After incubation with siramesine, all cells were stained at once with LysoTracker Green at a final concentration of $40 \mathrm{nM}$ and analysed with a flow cytometer. The geometric mean of LysoTracker Green fluorescence intensity for each sample is displayed. The experiments were performed in duplicate; the bars represent mean \pm S.D. (b) Fluorimetric detection of cytosolic and total cysteine cathepsin activity after siramesine treatment based on Z-FR-AMC substrate hydrolysis. HaCaT and U-87MG cells growing in a 96-well plate were treated with different siramesine concentrations. After incubation with siramesine, 15 or $200 \mu \mathrm{g} / \mathrm{ml}$ digitonin was added directly to the wells to lyse only the plasma membrane (cytosolic activity) or all cell membranes (total activity), respectively. After 12 min incubation with digitonin on ice, the cysteine cathepsin activity was measured fluorimetrically using Z-FR-AMC, and the initial velocities of the reactions were calculated. The experiments were performed in triplicate; the bars represent mean \pm S.D. (c) Western blotting and immunodetection of cathepsin L in cytosolic and total extracts of HaCaT cells $1 \mathrm{~h}$ after siramesine treatment. Cytosolic and total extracts were prepared with 15 or 30 , and $200 \mu \mathrm{g} / \mathrm{ml}$ of digitonin, respectively. Equal volumes of samples were loaded, proteins were resolved in $12.5 \%$ SDS-PAGE, transferred to a nitrocellulose membrane and labelled using specific antibodies. Z, Z-VAD-FMK; E, E-64d; T, $\alpha$-tocopherol; S, siramesine; D, digitonin; CYT, cytosolic; TOT, total 
a
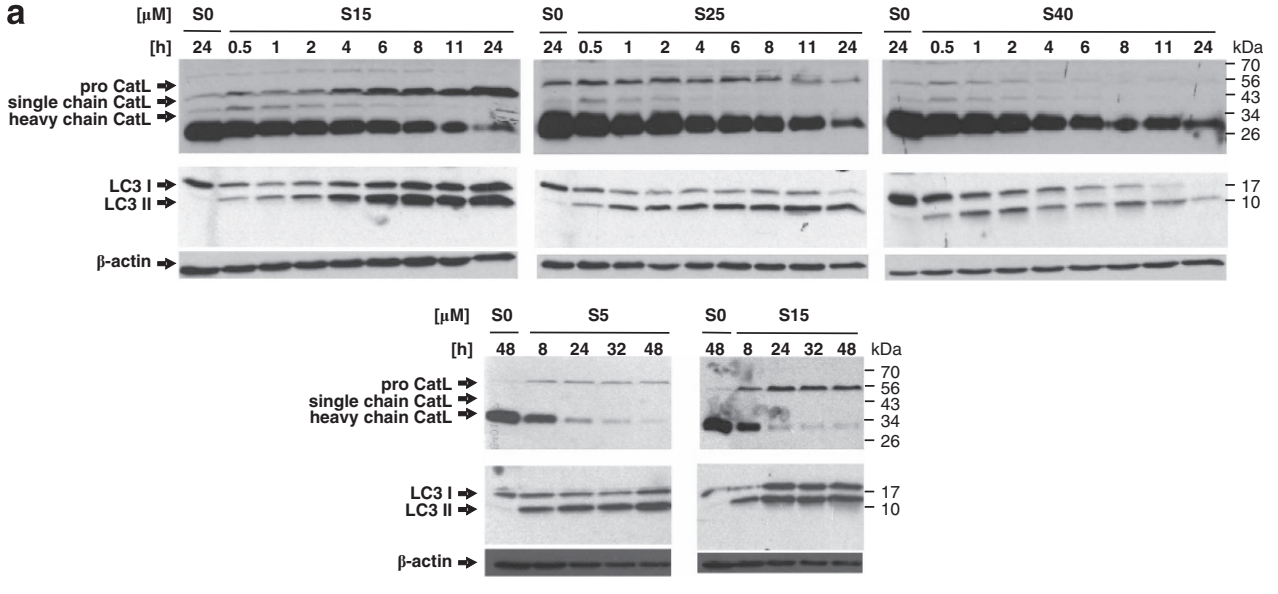

b
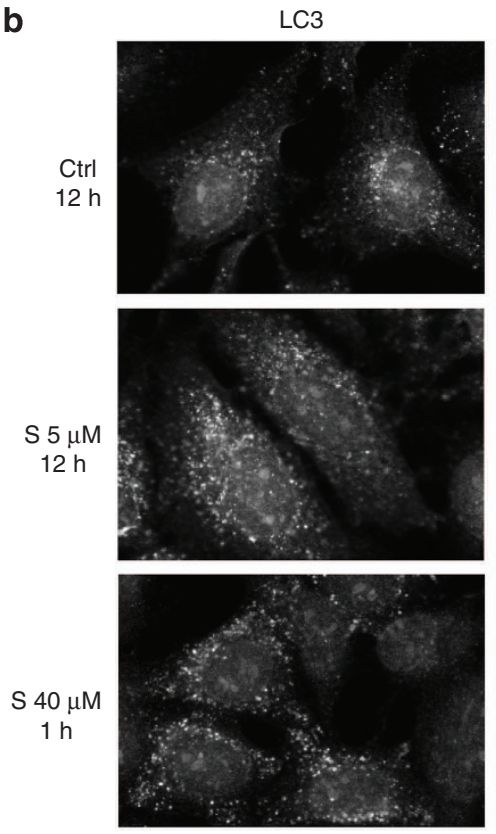

so
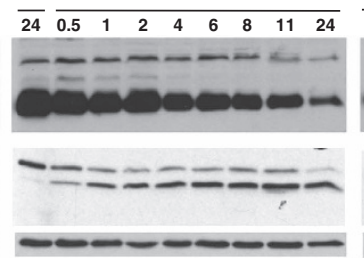

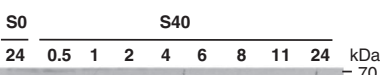
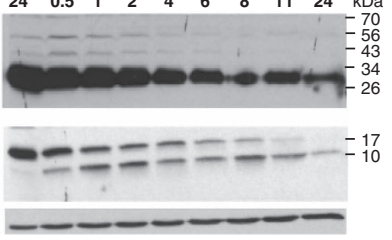

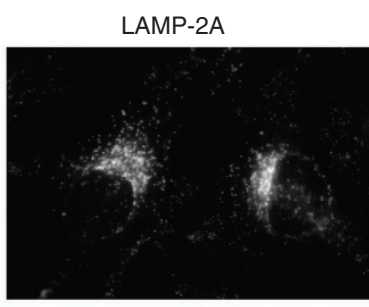

LC3 + LAMP-2A
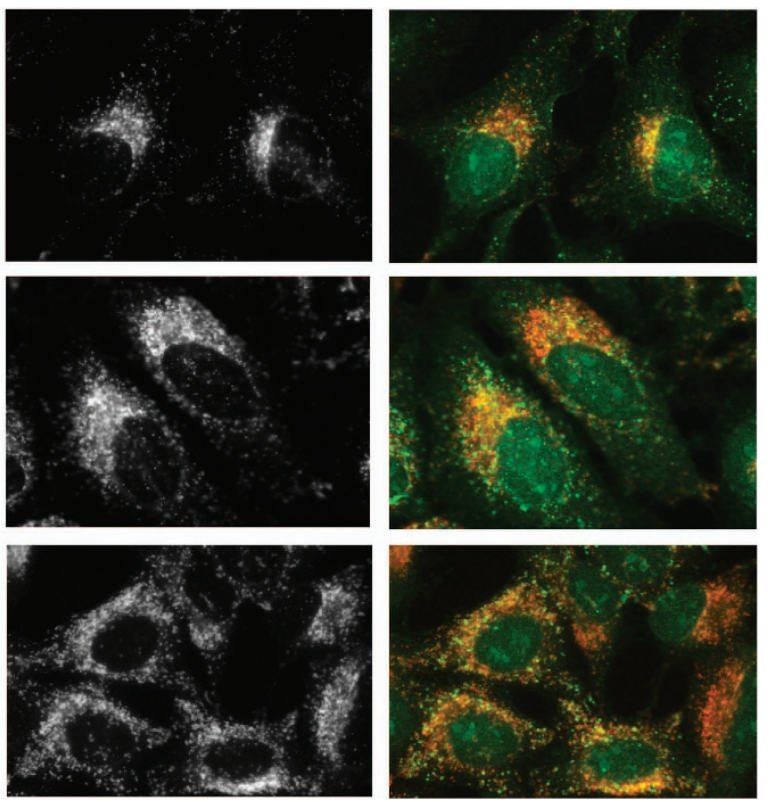

Figure 7 Siramesine-induced changes of cathepsin L and LC3 at a protein level. (a) HaCaT cell were treated with different siramesine concentrations, and the total cell extracts were prepared in RIPA buffer at the indicated time points. Proteins were resolved in $12.5 \%$ SDS-PAGE and transferred to a nitrocellulose membrane. Cathepsin L and LC3 were labelled with specific antibodies. (b) Immunocytochemistry of HaCaT cells treated with different concentrations of siramesine labelled with LC3 (green) and LAMP-2 A antibodies (red). S, siramesine

earlier cell studies. Along with detailed kinetic analyses of lysosomal and mitochondrial integrity, our study presents a comprehensive model of siramesine-induced cell death. Siramesine at concentrations above $20 \mu \mathrm{M}$ rapidly induces the loss of MMP, presumably by directly destabilising the mitochondrial membrane, and subsequently triggers apoptotic or non-apoptotic cell death, depending on the cell type. This can be probably explained by the promiscuity of siramesine. At siramesine concentrations below $20 \mu \mathrm{M}$ a slow cell death occurred, which was probably associated with progressive deterioration of cell homeostasis due to defects in multiple processes, including lysosomal degradation, autophagic flux, intracellular trafficking and energy metabolism. Importantly, siramesine-mediated cell death was found to be independent of LMP and, most probably, of sigma-2 receptors. Instead, siramesine exhibits a plethora of effects and the loss of mitochondrial integrity is the critical factor for the induction of cell death. Importantly, an agent with such a large range of targets is unlikely to exert its effect specifically on a particular subset of cells, such as cancer cells, thereby suggesting to re-evaluate the potential of siramesine and its analogues as drugs, and of sigma- 2 receptors as targets, in anticancer therapy.

\section{Materials and Methods}

Cell lines and reagents. Cell lines HeLa (human cervical carcinoma), MCF-7 (human breast carcinoma), Hsc-4 (human tongue squamous carcinoma), U-87MG (human glioblastoma-astrocytoma) and SH-SY5Y (human neuroblastoma) were purchased from the European Collection of Cell Cultures (Salisbury, UK). The HaCaT (human immortalised keratinocytes) cell line was purchased from the Cell Line Service GmbH (Eppelheim, Germany). All cells were tested for the absence of mycoplasma. Cell culture media DMEM, EMEM and Ham's F12/EMEM (1:1) and the supplements fetal bovine serum (FBS), penicillin/ streptomycin, stable L-glutamine, non-essential amino acids (NEAA) and sodium pyruvate were purchased from PAA (Pasching, Austria). Cathepsin inhibitor E-64d 


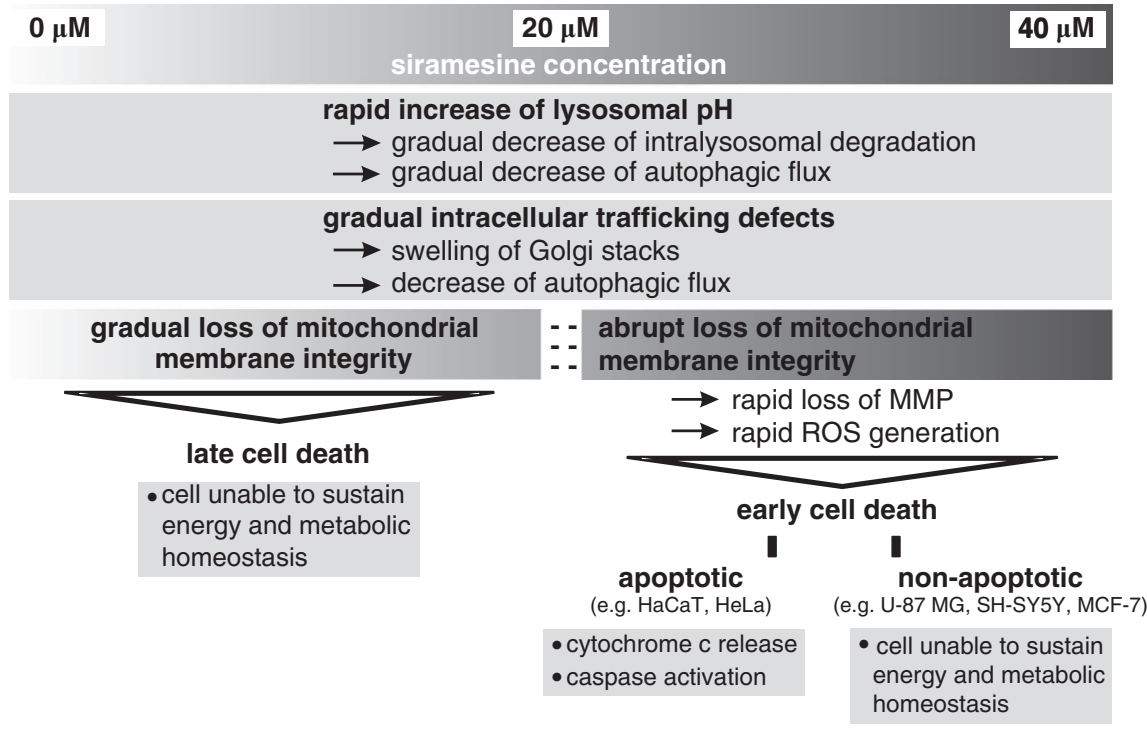

Figure 8 Schematic representation of siramesine-mediated effects on intracellular processes. All siramesine concentrations rapidly increase lysosomal pH and induce intracellular trafficking defects. Only high concentration (above $20 \mu \mathrm{M}$ ) trigger an abrupt loss of MMP, which leads to an early-onset cell death, which can be apoptotic or non-apoptotic, depending on the cell line

was purchased from Peptide Research Institute (Osaka, Japan). Caspase inhibitor Z-VAD-FMK was purchased from Bachem AG (Bubendorf, Switzerland). Antioxidants $\alpha$-tocopherol and NAC were purchased from Sigma-Aldrich (St. Louis, MO, USA). Siramesine was kindly provided by $\mathrm{H}$. Lundbeck A/S, Valby, Denmark.

For immunolabelling, the following antibodies were used: mouse mAb against cathepsin L, clone CPL33/1, (Abcam, Cambridge, UK) at 1:2500, mouse mAb against cytochrome $c$, clone 7H8.2C12 (BD Biosciences, Franklin Lakes, NJ, USA) at $1: 4000$, rabbit pAb against $L C 3$ (Abcam) at $1: 3000$; rabbit pAb against $\beta$-actin (Sigma-Aldrich) at 1:5000. All horseradish peroxidase-conjugated secondary antibodies was from Jackson ImmunoResearch (West Grove, PA, USA) and were used at $1: 5000$.

Cell culture and treatment. Cell lines HaCaT, HeLa and MCF-7 were cultured in DMEM supplemented with $10 \% \mathrm{FBS}, 2 \mathrm{mM}$ stable L-glutamine, $100 \mathrm{U} / \mathrm{ml}$ penicillin and $0.1 \mathrm{mg} / \mathrm{ml}$ streptomycin. Cell lines Hsc-4 and U-87MG were cultured in EMEM supplemented with 10\% FBS, 1\% NEAA, $1 \mathrm{mM}$ sodium pyruvate, $2 \mathrm{mM}$ stable L-glutamine, $100 \mathrm{U} / \mathrm{ml}$ penicillin and $0.1 \mathrm{mg} / \mathrm{ml}$ streptomycin The SH-SY5Y cell line was cultured in Ham's F12/EMEM (1:1), supplemented with $15 \% \mathrm{FBS}, 1 \% \mathrm{NEAA}, 2 \mathrm{mM}$ stable L-glutamine, $100 \mathrm{U} / \mathrm{ml}$ penicillin and $0.1 \mathrm{mg} / \mathrm{ml}$ streptomycin. All cells were cultured at $37^{\circ} \mathrm{C}$ in a humidified air atmosphere with $5 \% \mathrm{CO}_{2}$. The cells were seeded at least 1 day before the experiment.

Siramesine was dissolved in DMSO to make a $20 \mathrm{mM}$ stock solution, which was then diluted in complete culture medium to the final concentrations used. Inhibitors and antioxidants were applied $2 \mathrm{~h}$ before the siramesine treatment if not stated otherwise. E-64d was used at $10 \mu \mathrm{M}, \mathrm{Z}-\mathrm{VAD}-\mathrm{FMK}$ at $20 \mu \mathrm{M}, \alpha$-tocopherol at $0.3 \mathrm{mM}$ and NAC at $8 \mathrm{mM}$ final concentration. The final concentration of DMSO in the cell cultures did not exceed $2 \%$.

Cell viability assays. Cells were plated onto a 24 -well plate at $0.5 \times 10^{5}$ cells per well and grown overnight before the experiment. After $8 \mathrm{~h}$ of incubation with siramesine, cells were labelled with annexin V (BD Biosciences, Pharmingen, San Diego, CA, USA) and propidium iodide (Sigma-Aldrich) according to the manufacturer's instructions. The cells were then analysed using a FACSCalibur flow cytometer (BD Biosciences) and CellQuest software, v 3.3 (FACSComp Software; BD Biosciences). At least two independent experiments were performed, and each experiment was performed in duplicate.

Caspase activity measurements. HaCaT and U-87MG cells were plated in $6 \mathrm{~cm}$ petri dishes at $0.5 \times 10^{6}$ cells per dish and grown overnight before the siramesine treatment. Total cell extracts were prepared in the caspase buffer with detergents $(50 \mathrm{mM}$ HEPES, $200 \mathrm{mM} \quad \mathrm{NaCl}, \quad 10 \%$ (w/v) sucrose, $0.1 \%$ (w/v) CHAPS, $5 \mathrm{mM} \mathrm{MgCl}, 0.02 \%$ (w/v) bovine serum albumin (BSA), $1 \%(\mathrm{w} / \mathrm{v})$ NP-40, $0.5 \%(\mathrm{w} / \mathrm{v})$ Triton X-100, $\mathrm{pH} 7.5)$ and sonicated for $5 \mathrm{~s}$. Samples were then centrifuged at $16100 \times g$ for $10 \mathrm{~min}$ at $4^{\circ} \mathrm{C}$, and the supernatant was stored at $-80^{\circ} \mathrm{C}$. After determining the protein concentration with a Bradford reagent (Bio-Rad, Hercules, CA, USA), $75 \mu \mathrm{g}$ of protein was transferred to a 96-well plate and caspase buffer without detergents was added to a final volume of $90 \mu$ l. After 10-min incubation at $37^{\circ} \mathrm{C}, 10 \mu$ fluorogenic substrate Ac-DEVD-AFC (Bachem AG) was added (final concentration of $10 \mu \mathrm{M}$ ), followed by continuous measurements of substrate hydrolysis for at least $30 \mathrm{~min}$ at excitation and emission wavelengths of 400 and $505 \mathrm{~nm}$, respectively, using a Tecan Saphire microplate reader (Tecan, Männedorf, Switzerland). The initial velocities of the reactions were calculated and are presented in bar plots. Three independent experiments were performed, and each experiment was performed in triplicate.

Determination of lysosomal and mitochondrial membrane integrity by flow cytometry. HaCaT and U-87MG cells were plated onto a 24-well plate at $0.5 \times 10^{5}$ cells per well and grown overnight before the siramesine treatment. After incubation with siramesine, the lysosomal and mitochondrial membrane integrity were measured based on the uptake of LysoTracker Green DND 26 (Invitrogen, Molecular Probes, Eugene, OR, USA), JC-1 (Enzo Life Sciences, Farmingdale, NY, USA) or NAO (Invitrogen, Molecular Probes), respectively. LysoTracker Green DND 26 was added at a final concentration of $40 \mathrm{nM}$ and incubated for $10 \mathrm{~min}$. JC-1 was added for $15 \mathrm{~min}$ at a final concentration of $3.5 \mu \mathrm{g} / \mathrm{ml}$, whereas NAO was added at a final concentration of $50 \mathrm{nM}$ and incubated for $15 \mathrm{~min}$. After the staining, cells were washed and detached using TrypLE Select (Gibco, Life Technologies, Carlsbad, CA, USA), the samples were then analysed with a FACSCalibur flow cytometer and CellQuest software, $v$ 3.3. The values for the geometric mean of fluorescence intensity were read for LysoTracker Green, and percentages of bright and pale cells were read for JC-1, whereas histograms are shown for NAO staining. At least two independent experiments were performed, and each experiment was performed in duplicate.

Cathepsin activity measurements. HaCaT and U-87MG cells were plated onto a 96-well plate at $0.8 \times 10^{4}$ cells per well and grown overnight before the siramesine treatment. After 1-h incubation with siramesine, cells were washed with PBS buffer (PAA), followed by the addition of different concentrations of digitonin (Sigma-Aldrich; 15, 30, 45, 60, 70, $200 \mu \mathrm{g} / \mathrm{ml}$ ) in acetate buffer $(50 \mathrm{mM}$ Na-acetate, $150 \mathrm{mM} \mathrm{NaCl}, 0.5 \mathrm{mM}$ EDTA, $\mathrm{pH} 5.6$ ) to lyse the plasma membrane only (cytosolic activity) or all cell membranes (total activity). After 12 min incubation with digitonin on ice, the cysteine cathepsin activity was measured fluorimetrically 
using Z-FR-AMC (Bachem AG) as the substrate at a final concentration of $30 \mu \mathrm{M}$ in the presence of $5 \mathrm{mM}$ DTT. Substrate hydrolysis was measured at excitation and emission wavelengths of 380 and $460 \mathrm{~nm}$, respectively, for at least 30 min using a Tecan Saphire microplate reader. The initial velocities of the reactions were calculated and are presented as bar plots. Three independent experiments were performed, and each experiment was performed in triplicate.

Preparation of cytosolic and total extracts with digitonin. HaCaT and U-87MG cells were plated in $10 \mathrm{~cm}$ petri dishes at $1.5 \times 10^{6}$ cells per dish and grown overnight before the siramesine treatment. Two hours before the treatment, $\alpha$-tocopherol was added at a final concentration of $0.3 \mathrm{mM}$. After incubation with siramesine, the culture supernatant containing detached cells were transferred into a tube. Next, the attached cells were detached by TrypLE Select, collected and transferred into the same tube. The cells were then spun down, and the cytosolic and total extracts were prepared from the cell pellets using digitonin at 15,30 or $200 \mu \mathrm{g} / \mathrm{ml}$, respectively. The samples were incubated with digitonin for $10 \mathrm{~min}$ on ice and then spun down at $1000 \times g$ for $5 \mathrm{~min}$ at $4^{\circ} \mathrm{C}$.

Preparation of total extracts in RIPA buffer. HaCaT and U-87MG cells were plated in $10 \mathrm{~cm}$ petri dishes at $1.5 \times 10^{6}$ cells per dish and grown overnight before the siramesine treatment. After incubation with siramesine, the culture supernatant containing detached cells was transferred into a tube, spun down and the pelleted cells were lysed with RIPA buffer $(50 \mathrm{mM}$ Tris/ $\mathrm{HCl}, 100 \mathrm{mM}$ $\mathrm{NaCl}, 0.1 \%$ (w/v) SDS, $1 \%$ (w/v) NP-40, 0.5\% (w/v) deoxycholic acid, $1 \mathrm{mM}$ EDTA, $\mathrm{pH}$ 8.0). The attached cells were lysed in the plate and collected with a cell scraper. The lysates of detached and attached cells were combined, sonicated for $5 \mathrm{~s}$ and centrifuged at $16100 \times \mathrm{g}$ for $10 \mathrm{~min}$ at $4^{\circ} \mathrm{C}$.

Protein analysis. The protein concentration in the cell extracts was determined with a Bradford reagent (Bio-Rad). Equal amounts of protein were loaded and resolved in $15 \%$ or $12.5 \%$ SDS-PAGE gels and electrotransferred to nitrocellulose membranes. For blocking non-specific interactions, the membranes were incubated in $5 \%$ skimmed milk in TBS $(20 \mathrm{mM}$ Tris/ $/ \mathrm{HCl}, 0.5 \mathrm{M} \mathrm{NaCl}, \mathrm{pH} 7.5)$ for at least $1 \mathrm{~h}$. The membranes were incubated overnight with primary antibodies and for $2 \mathrm{~h}$ with secondary antibodies. The proteins were then visualised with $\mathrm{ECL}$ (Amersham Biosciences, Piscataway, NJ, USA), according to the manufacturer's instructions. To confirm equal protein loading, all immunoblots were also probed with $\beta$-actin. All experiments were repeated at least three times.

Measurements of ROS. HaCaT cells and U-87MG were plated onto a 24-well plate at $0.5 \times 10^{5}$ cells per well and grown overnight before the siramesine treatment. Two hours before the treatment, $\alpha$-tocopherol was added at a final concentration of $0.3 \mathrm{mM}$. After incubation with siramesine, cells were washed with PBS and incubated with $5 \mu \mathrm{M} \mathrm{CM}-\mathrm{H}_{2} \mathrm{DCFDA}$ (Invitrogen, Molecular Probes) in PBS for $40 \mathrm{~min}$. The cells were then detached using TrypLE Select and analysed at once with a FACSCalibur flow cytometer and CellQuest software, v 3.3. The values for the geometric mean of fluorescence intensity were read. At least two independent experiments were performed, and each experiment was performed in duplicate. Because of the technical constraints, we were not able to monitor the formation of ROS at high siramesine concentration at longer times points when a significant part of the cells had already detached from the surface.

Immunocytochemistry. HaCaT cells were grown overnight on coverslips after coating them with poly-L-lysine (Sigma-Aldrich). On the next day, the cells were treated with siramesine and incubated for the indicated time points. After incubation, the cells were fixed in cold methanol for $13 \mathrm{~min}$ on ice. The coverslips were washed with PBS, followed by the addition of $3 \%$ BSA in PBS for 30 min. Primary antibodies were diluted in 3\% BSA (rabbit pAb against LC3 (Abcam) at 1:1000, mouse mAb against LAMP-2 A, clone H4B4, (Abcam) at 1:100) and incubated for $2 \mathrm{~h}$ at room temperature. After incubation, the coverslips were washed with PBS and secondary antibodies (rabbit green, mouse red at 1:1000 (Invitrogen, Molecular Probes)) were added for 1.5-h incubation at room temperature. After washing with PBS, the ProLong Gold antifade reagent (Invitrogen, Molecular Probes) was added to the glass slide and covered with a coverslip. The samples were then observed using an Olympus IX81 microscope (Tokyo, Japan).

TEM and Epon embedding. HaCaT and U-87MG cells were plated in $10 \mathrm{~cm}$ petri dishes at $1.5 \times 10^{6}$ cells per dish and grown overnight before the siramesine treatment. Two hours before the treatment, $\alpha$-tocopherol was applied at a final concentration of $0.3 \mathrm{mM}$. For the analysis of ultrastructure, the cells were embedded in epoxy resin. At the end of the incubation with siramesine, the cells were fixed by adding $2 \%$ glutaraldehyde (GA; Sigma-Aldrich) in $200 \mathrm{mM}$ HEPES buffer to the culture medium at a $1: 1$ volume ratio. After $15 \mathrm{~min}$, the fixative containing the detached cells was transferred to a tube, whereas the attached cells were added after being scraped. The cells were spun down, resuspended in fresh $1 \% \mathrm{GA}$ and incubated for $3 \mathrm{~h}$ at room temperature. For epoxy resin embedding, the samples were postfixed with $2 \% \mathrm{OsO}_{4}$ (EMS, Hatfield, PA, USA) solution containing $1.5 \%$ potassium ferricyanide for $1 \mathrm{~h}$, and stained en bloc with $1.5 \%$ aqueous uranyl acetate (EMS) for $30 \mathrm{~min}$. The cells were then dehydrated using a graded ethanol series and embedded in epoxy resin (Sigma-Aldrich). Ultrathin sections of $60-70 \mathrm{~nm}$ were cut with an ultramicrotome (Leica Ultracut UCT, Leica Microsystems, Wetzlar, Germany) and examined using a Philips CM100 transmission electron microscope (Philips, Eindhoven, The Netherlands). The images were recorded digitally with a Quemsa TEM CCD camera (Olympus Soft Imaging Solutions, Muenster, Germany) and iTEM software (Olympus Soft Imaging Solutions).

Stereological analysis of the presence of the Golgi cisternae in U-87MG cells. For each sample, random parts of a thin section were systematically sampled at $\times 900$ magnification in order to estimate the cytoplasmic area (volume). At least 30 profiles of different cells were included in the analysis. Within these areas, all identifiable Golgi cisternae were imaged at $\times 8900$ magnification. To analyse the micrographs, a stereological test grid with horizontal and vertical lines was used. To estimate the total volume of the cytoplasm, which represented a reference space, the number of test points over the cytoplasm was counted. For the analysis of the presence of Golgi cisternae, the number of intersections of all identifiable Golgi cisternal membrane with horizontal test lines was counted in order to estimate the total length of the Golgi cisternal membrane. A cisterna was defined as an elongated enclosed membrane profile with the length minimum twice its breadth and the breadth $<100 \mathrm{~nm}$. From these values, the ratios of the total number of intersections (length) of the Golgi cisternal membranes to the total volume of cytoplasm were calculated, representing relative surface density of the Golgi cisternae.

\section{Conflict of Interest}

The authors declare no conflict of interest.

Acknowledgements. The work was supported by grants P1-0140, J1-4121 and J1-3602 from the Slovene Research Agency. We thank H Lundbeck A/S, Valby, Denmark, for a generous gift of siramesine; Gareth Griffiths for inspiring discussion and help with the interpretation of EM micrographs; John Lucocq for advice on stereological analysis of the presence of Golgi stacks; the Electron Microscopy Unit for Biological Sciences, Department of Biosciences, University of Oslo; and Vladyslava Hostyeva for her technical assistance with EM analysis.

1. Martin WR, Eades CG, Thompson JA, Huppler RE, Gilbert PE. The effects of morphineand nalorphine- like drugs in the nondependent and morphine-dependent chronic spinal dog. J Pharmacol Exp Ther 1976; 197: 517-532.

2. Quirion R, Bowen WD, Itzhak Y, Junien JL, Musacchio JM, Rothman RB et al. A proposal for the classification of sigma binding sites. Trends Pharmacol Sci 1992; 13: 85-86.

3. Hellewell SB, Bowen WD. A sigma-like binding site in rat pheochromocytoma (PC12) cells: decreased affinity for $(+)$-benzomorphans and lower molecular weight suggest a different sigma receptor form from that of guinea pig brain. Brain Res 1990; 527: 244-253.

4. Colabufo NA, Berardi F, Contino M, Niso M, Abate C, Perrone R et al. Antiproliferative and cytotoxic effects of some sigma2 agonists and sigma1 antagonists in tumour cell lines. Naunyn Schmiedebergs Arch Pharmacol 2004; 370: 106-113.

5. Spruce BA, Campbell LA, McTavish N, Cooper MA, Appleyard MV, O'Neill M et al. Small molecule antagonists of the sigma-1 receptor cause selective release of the death program in tumor and self-reliant cells and inhibit tumor growth in vitro and in vivo. Cancer Res 2004; 64: $4875-4886$.

6. Crawford KW, Bowen WD. Sigma-2 receptor agonists activate a novel apoptotic pathway and potentiate antineoplastic drugs in breast tumor cell lines. Cancer Res 2002; 62: 313-322.

7. Hornick JR, Vangveravong S, Spitzer D, Abate C, Berardi F, Goedegebuure P et al. Lysosomal membrane permeabilization is an early event in sigma-2 receptor ligand mediated cell death in pancreatic cancer. J Exp Clin Cancer Res 2012; 31: 41. 
8. Jonhede S, Petersen A, Zetterberg M, Karlsson JO. Acute effects of the sigma-2 recepto agonist siramesine on lysosomal and extra-lysosomal proteolytic systems in lens epithelial cells. Mol Vis 2010; 16: 819-827.

9. Ostenfeld MS, Fehrenbacher N, Hoyer-Hansen M, Thomsen C, Farkas T, Jaattela M. Effective tumor cell death by sigma-2 receptor ligand siramesine involves lysosoma leakage and oxidative stress. Cancer Res 2005; 65: 8975-8983.

10. Zeng C, Rothfuss J, Zhang J, Chu W, Vangveravong S, Tu Z et al. Sigma-2 ligands induce tumour cell death by multiple signalling pathways. Br J Cancer 2012; 106 693-701.

11. Bem WT, Thomas GE, Mamone JY, Homan SM, Levy BK, Johnson FE et al. Overexpression of sigma receptors in nonneural human tumors. Cancer Res 1991; 51: 6558-6562.

12. Vilner BJ, John CS, Bowen WD. Sigma-1 and sigma-2 receptors are expressed in a wide variety of human and rodent tumor cell lines. Cancer Res 1995; 55 408-413

13. Walker JM, Bowen WD, Walker FO, Matsumoto RR, De Costa B, Rice KC. Sigma receptors: biology and function. Pharmacol Rev 1990; 42: 355-402.

14. Banister SD, Kassiou M. The therapeutic potential of sigma (sigma) receptors for the treatment of central nervous system diseases: evaluation of the evidence. Curr Pharm Des 2012; 18: 884-901.

15. Heading C. Siramesine H Lundbeck. Curr Opin Investig Drugs 2001; 2: 266-270.

16. Moltzen EK, Perregaard J, Meier E. Sigma ligands with subnanomolar affinity and preference for the sigma 2 binding site. 2. Spiro-joined benzofuran, isobenzofuran, and benzopyran piperidines. J Med Chem 1995; 38: 2009-2017.

17. Perregaard J, Moltzen EK, Meier E, Sanchez C. Sigma ligands with subnanomolar affinity and preference for the sigma 2 binding site. 1. 3-(omega-aminoalkyl)-1 $\mathrm{H}$-indoles. J Med Chem 1995; 38: 1998-2008.

18. Soby KK, Mikkelsen JD, Meier E, Thomsen C. Lu 28-179 labels a sigma(2)-site in rat and human brain. Neuropharmacology 2002; 43: 95-100.

19. Hornick JR, Xu J, Vangveravong S, Tu Z, Mitchem JB, Spitzer D et al. The novel sigma-2 receptor ligand SW43 stabilizes pancreas cancer progression in combination with gemcitabine. Mol Cancer 2010; 9: 298.

20. Ostenfeld MS, Hoyer-Hansen M, Bastholm L, Fehrenbacher N, Olsen OD, Groth-Pedersen L et al. Anti-cancer agent siramesine is a lysosomotropic detergent that induces cytoprotective autophagosome accumulation. Autophagy 2008; 4: 487-499.

21. Spirkoski J, Melo FR, Grujic M, Calounova G, Lundequist A, Wernersson S et al. Mast cell apoptosis induced by siramesine, a sigma-2 receptor agonist. Biochem Pharmacol 2012; 84: $1671-1680$

22. Cirman T, Oresic K, Droga-Mazovec G, Turk V, Reed JC, Myers RM et al. Selective disruption of lysosomes in HeLa cells triggers apoptosis mediated by cleavage of Bid by multiple papain-like lysosomal cathepsins. J Biol Chem 2004; 279 3578-3587.

23. Droga-Mazovec G, Bojič L, Petelin A, Ivanova S, Romih R, Repnik U et al. Cysteine cathepsins trigger caspase-dependent cell death through cleavage of bid and antiapoptotic Bcl-2 homologues. J Biol Chem 2008; 283: 19140-19150.

24. Grau A, Ortiz A. Dissimilar protection of tocopherol isomers against membrane hydrolysis by phospholipase A2. Chem Phys Lipids 1998; 91: 109-118.

25. Sadowska AM, Manuel YKB, De Backer WA. Antioxidant and anti-inflammatory efficacy of NAC in the treatment of COPD: discordant in vitro and in vivo dose-effects: a review. Pulm Pharmacol Ther 2007; 20: 9-22.

26. Ivanova S, Repnik U, Bojič L, Petelin A, Turk V, Turk B. Lysosomes in apoptosis. Methods Enzymol 2008; 442: 183-199.
27. Klionsky DJ, Abdalla FC, Abeliovich $\mathrm{H}$, Abraham RT, Acevedo-Arozena A, Adeli K et al. Guidelines for the use and interpretation of assays for monitoring autophagy. Autophagy 2012; 8: 445-544.

28. Zeng C, Vangveravong S, Xu J, Chang KC, Hotchkiss RS, Wheeler KT et al. Subcellular localization of sigma-2 receptors in breast cancer cells using two-photon and confocal microscopy. Cancer Res 2007; 67: 6708-6716.

29. Abate C, Perrone R, Berardi F. Classes of sigma2 (sigma2) receptor ligands: structure affinity relationship (SAfiR) studies and antiproliferative activity. Curr Pharm Des 2012; 18: 938-949.

30. Mamolo MG, Zampieri D, Zanette C, Florio C, Collina S, Urbano M et al. Substituted benzylaminoalkylindoles with preference for the sigma2 binding site. Eur $\mathrm{J}$ Med Chem 2008; 43: 2073-2081.

31. Kagan VE, Tyurin VA, Jiang J, Tyurina YY, Ritov VB, Amoscato AA et al. Cytochrome $c$ acts as a cardiolipin oxygenase required for release of proapoptotic factors. Nat Chem Biol 2005; 1: 223-232.

32. Turk B, Turk V. Lysosomes as "suicide bags" in cell death: myth or reality? J Biol Chem 2009; 284: 21783-21787.

33. Repnik U, Hafner Česen M, Turk B. The endolysosomal system in cell death and survival. Cold Spring Harb Perspect Biol 2013; 5: a008755

34. Repnik U, Stoka V, Turk V, Turk B. Lysosomes and lysosomal cathepsins in cell death. Biochim Biophys Acta 2012; 1824: 22-33

35. Vilner BJ, Bowen WD. Modulation of cellular calcium by sigma-2 receptors: release from intracellular stores in human SK-N-SH neuroblastoma cells. J Pharmacol Exp Ther 2000; 292: $900-911$

36. Parry MJ, Alakoskela JM, Khandelia H, Kumar SA, Jaattela M, Mahalka AK et al. High-affinity small molecule-phospholipid complex formation: binding of siramesine to phosphatidic acid. J Am Chem Soc 2008; 130: 12953-12960.

37. Kooijman EE, Chupin V, de Kruijff B, Burger KN. Modulation of membrane curvature by phosphatidic acid and lysophosphatidic acid. Traffic 2003; 4: 162-174.

38. Kagan VE, Bayir HA, Belikova NA, Kapralov O, Tyurina YY, Tyurin VA et al. Cytochrome c/cardiolipin relations in mitochondria: a kiss of death. Free Radic Biol Med 2009; 46: 1439-1453.

39. Yang SB, Proks P, Ashcroft FM, Rupnik M. Inhibition of ATP-sensitive potassium channels by haloperidol. Br J Pharmacol 2004; 143: 960-967.

40. Cassano G, Gasparre G, Contino M, Niso M, Berardi F, Perrone R et al. The sigma-2 receptor agonist PB28 inhibits calcium release from the endoplasmic reticulum of SK-N-SH neuroblastoma cells. Cell Calcium 2006; 40: 23-28.

41. Monassier L, Manoury B, Bellocq C, Weissenburger J, Greney H, Zimmermann D et al. sigma(2)-receptor ligand-mediated inhibition of inwardly rectifying $\mathrm{K}(+)$ channels in the heart. J Pharmacol Exp Ther 2007; 322: 341-350

42. Muench SP, Trinick J, Harrison MA. Structural divergence of the rotary ATPases. $Q$ Rev Biophys 2011; 44: 311-356

43. Nakanishi-Matsui M, Sekiya M, Nakamoto RK, Futai M. The mechanism of rotating proton pumping ATPases. Biochim Biophys Acta 2010; 1797: 1343-1352.

(c) (2) (2) Cell Death and Disease is an open-access journa published by Nature Publishing Group. This work is licensed under a Creative Commons Attribution-NonCommercialShareAlike 3.0 Unported License. To view a copy of this license, visit http://creativecommons.org/licenses/by-nc-sa/3.0/

\section{Supplementary Information accompanies this paper on Cell Death and Disease website (http://www.nature.com/cddis)}

